\title{
The protracted TB struggle - SA ups the intensity
}

An ambitious programme to integrate TB and HIV services into single primary health care facilities and a R32 million European Union (EU)-funded treatment defaulter tracing project are among the chief mitigation weapons aimed at the TB pandemic this year. This emerged from an interview last month with the national director of the TB Control and Management Unit at the national health department, Dr Lindiwe Mvusi. patient out there. Currently we pick them up passively by waiting until they come into a facility, which can so often be tragically late, let alone ineffective.'

Last June Government took over the funding of a R32 million treatment defaulter (and contact) tracing and treatment support programme from the EU. It was determined to strengthen community care - even mooting the possibility of treating multidrug-

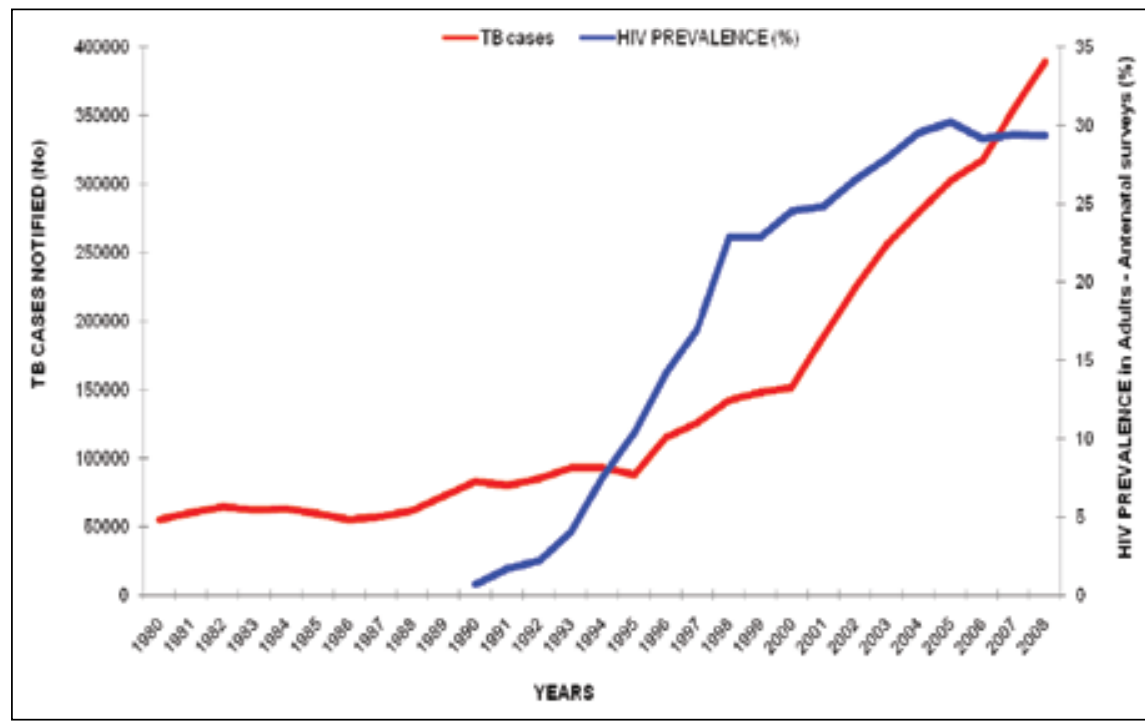

Fig. 1. TB/HIV case/prevalence comparison (source: Stats SA).

Table I. Provincial comparisons of TB incidence and antenatal clinic HIV prevalence with relevant provincial population estimates (source: Stats SA).

\begin{tabular}{|l|c|c|c|}
\hline Province & $\begin{array}{l}\text { Population 2009 } \\
\text { mid-yr estimates }\end{array}$ & $\begin{array}{l}\text { TB incidence } \\
2008\end{array}$ & $\begin{array}{l}\text { ANC HIV } \\
\text { Prevalance (\%) }\end{array}$ \\
\hline EC & 6648600 & 965.5 & 27.6 \\
FS & 2902400 & 889.6 & 32.9 \\
GP & 10531300 & 465.6 & 29.9 \\
KZN & 10449300 & 1167.8 & 38.7 \\
LP & 5227200 & 421.0 & 20.7 \\
MP & 3606800 & 629.6 & 35.5 \\
NC & 1447600 & 935.1 & 16.2 \\
NW & 3450400 & 798.2 & 31.0 \\
WC & 5356900 & 953.8 & 16.1 \\
\hline
\end{tabular}

With the HIV pandemic having multiplied South Africa's TB prevalence three-fold over the past decade (Fig. 1) and the country now among the 10 worst performing in TB control internationally, Mvusi says more efficient awareness, prevention and monitoring campaigns are 'crucial'. 'We need to work harder at getting to the resistant (MDR) patients in the community.

Some 72 teams (each with a retired enrolled nurse, 2 community health nurses and a data capturer) working in as many health sub-districts, covering 726 health facilities, are doing defaulter tracing based on facility lists of patients who miss treatment, 'even for a day or two'. 'We find out what their issues are, counsel them and get them back for treatment - re-treatment patients who have to get daily injections of streptomycin are given these at home instead of travelling to the clinic at own cost,' she added.

This project resulted in a decrease in the defaulter rate wherever it was implemented. Explained Mvusi: 'There's even talk of managing MDR patients in the community and we're thinking it could be added onto this current programme, but we don't want to overwhelm our teams - we'd rather increase our case findings, and decrease our defaulters and loss to follow-up first'.

'We find out what their
issues are, counsel them and
get them back for treatment
- re-treatment patients who
have to get daily injections
of streptomycin are given
these at home instead of
travelling to the clinic at
own cost,' she added.

\section{Applying knowledge effectively}

The other intervention with the potential for making a major difference this year when it will be significantly expanded is the Palsa-Plus (University of Cape Town Lung Institute-designed) programme aimed at early diagnosis of lung diseases, including TB, and effectively managing TB/HIV co-infected patients. (Palsa $=$ Practical Approach to Lung Health in South Africa). It was piloted in the Free State in 2004, where clinics reported $70 \%$ improved TB detection and $80 \%$ improvement in asthma management since their nurses started the basic Palsa training. Based on these findings the Free State then scaled it up throughout the province, and it was later implemented in the Western Cape (see Table I for provincial TB incidence/HIV prevalence).

This approach of on-site health care worker training, using evidence-based 


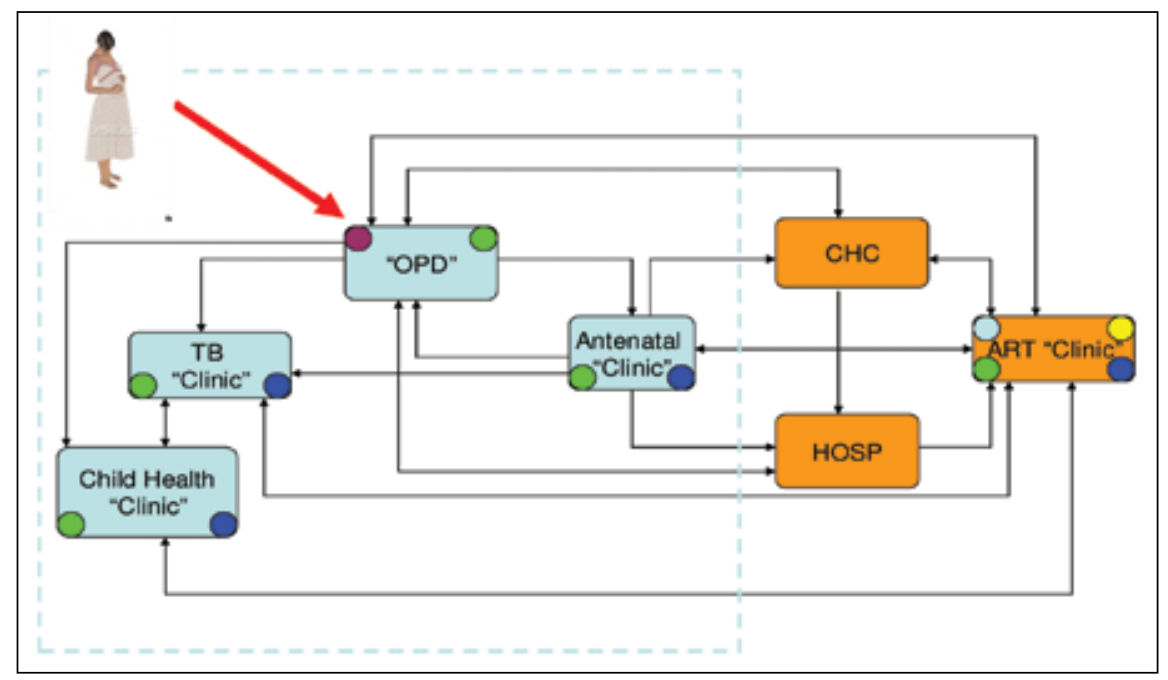

Fig. 2. Challenges and barriers to care (source: National Department of Health).

guidelines, that integrates content with process began in the high TB burden health districts of Ethekwini (Durban), Nelson Mandela (Port Elizabeth), Amathole (East London) and City of Johannesburg at the beginning of last year.

The strategy includes pre-ART care for all HIV-positive patients, prevention of TB and other opportunistic infections, prevention of mother-to-child infection, prevention and treatment of sexually transmitted diseases, cervical cancer screening, drug adherence counselling, health maintenance and appropriate referral for ART. It serves as a foundation for consolidating uncomplicated ART-eligible adults to primary health care facilities where appropriately trained nurses can treat them, thus reserving the scarcer doctors for children and other more complex cases. There are also many challenges and barriers to care experienced by pregnant women in the current unintegrated service provision model, said Mvusi. This often led to loss to follow-up, with patients not getting the necessary care, suffering unnecessary complications and dying (Fig. 2).

\section{Online Children and TB training manual}

Mvusi said another major intervention will be contact tracing and a doctor training campaign that will focus on lessening the huge TB case load among children, who are notoriously difficult to diagnose, especially when co-infected with HIV.

This training will be available online 'very soon' in the form of an electronic training manual written by Professors Robert Gie and Robin Wood (Stellenbosch and Cape Town universities, respectively), both

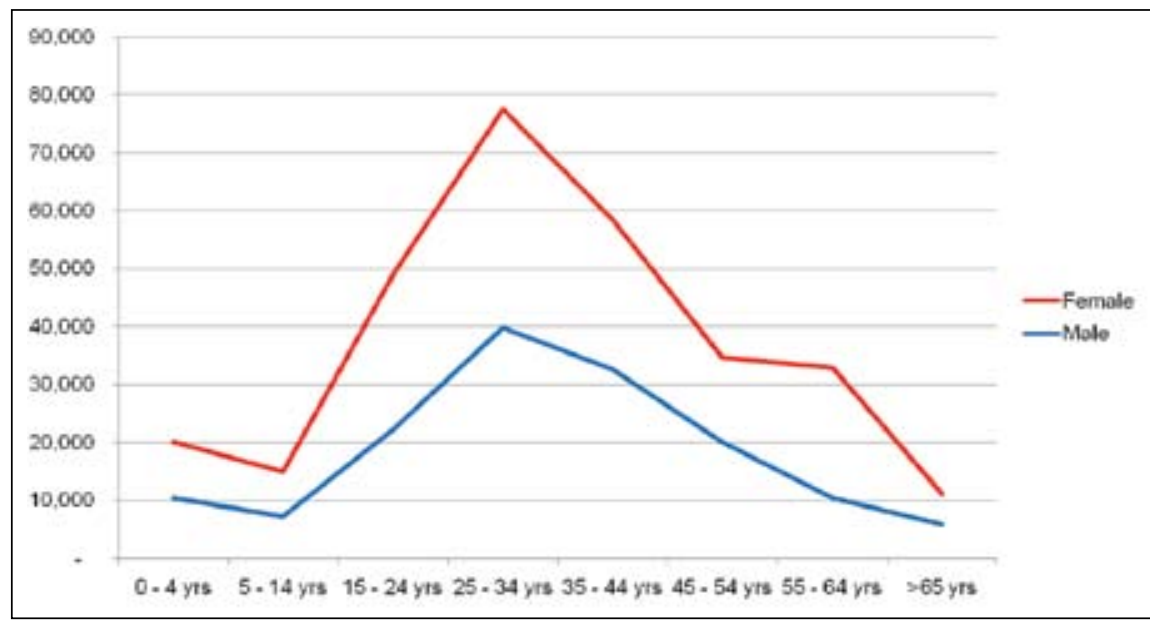

Fig. 3. Graph showing low numbers of children under 5 years of age with TB, pointing to low case detection among children (source: National Department of Health).

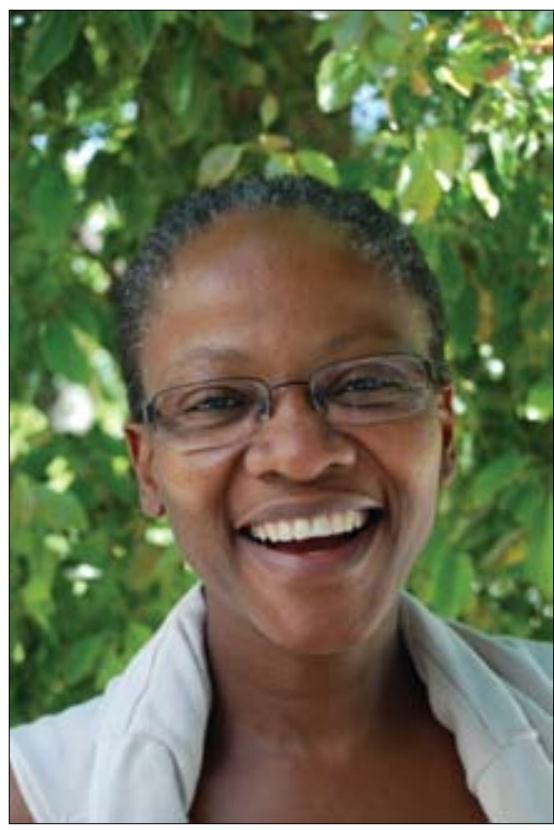

Dr Lindiwe Mvusi, National Director of the TB Control and Management Unit at the national department of health.

\section{Picture: Chris Bateman}

leaders in their fields. This is aimed at improving diagnostic skills among doctors as there is under-diagnosis of TB in children, with increasing mortality (Fig. 3). (The Stats SA 2009 report on 'Mortality and causes of death in South Africa' states that TB is the fourth highest cause of death in children between 1 and 4 years of age.) The intervention will be accompanied by an intensified contact tracing programme to ensure that all close contacts of people diagnosed with infectious TB are screened and tested for TB - mainly children under 5 years and people who are HIV positive. All asymptomatic children less than 5 years and HIVpositive clients will be given TB preventive therapy to reduce morbidity in these groups.

Considering the high costs of treating drug-resistant $T B$, it is critical to prevent the transmission of MDR and XDR TB.

\section{MDR and XDR TB}

Asked to give an update on the national MDR, XDR TB situation, Mvusi said that after the dramatic 2006 Tugela Ferry outbreak in KwaZulu-Natal a thorough survey of 10 other hospitals 


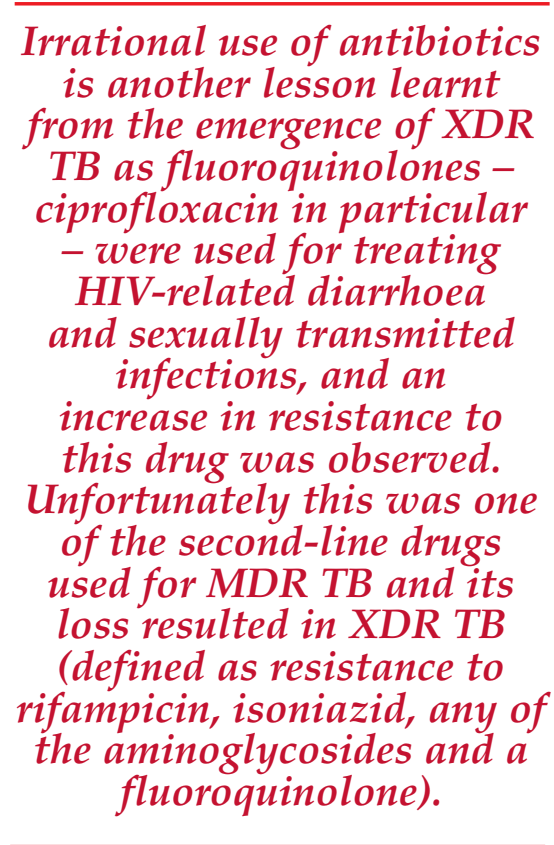

in the province revealed no similar 'outbreaks'. Retrospective testing of all stored isolates and routine testing of all MDR TB patients and suspected MDR TB patients in the country's 21 TB hospitals resulted in an increase in the numbers of MDR and XDR TB diagnosed, but nothing that justified the widespread media alarm (Table II). The prevalence of XDR TB among MDR TB patients is $9 \%$ - in line with the global trend - but the numbers are among the highest in the world.

The 4 provinces with the highest burden of MDR and XDR TB are KwaZulu-Natal, Eastern Cape, Western Cape and Gauteng. Considering the high costs of treating drug-resistant TB,

Table II. MDR/XDR TB statistics from 2004 to 2008 indicating how costly a failure to contain such transmission could become (source: National Department of Health)

\begin{tabular}{|l|r|r|r|r|r|}
\hline & 2004 & 2005 & 2006 & 2007 & 2008 \\
MDR TB & 3278 & 4305 & 6716 & 3757 & 4535 \\
XDR TB & 74 & 184 & 419 & 493 & 418 \\
Total & 3352 & 4489 & 7135 & 4250 & 4953 \\
\hline
\end{tabular}

it is critical to prevent the transmission of MDR and XDR TB.

Infection control emerged as a major problem in hospitals, so guidelines were widely disseminated and health care staff training upgraded to include airborne infection control. 'We widened the training, through the CSIR, to include maintenance managers, architects and engineers so that ventilation, fans and UV lights were optimally used, and ensure that with the new hospitals being built airborne infection control measures are taken into consideration.' Where facility infrastructure limited the implementation of optimal infection control measures, patients were rather made to wait outside in the open air under shelter and surgical masks issued once they were inside the clinic or hospital. Mvusi said a much more holistic approach needs to be taken on TB prevention, with minimum norms and standards for health facilities and housing currently being drawn up by the CSIR.

Irrational use of antibiotics is another lesson learnt from the emergence of XDR TB as fluoroquinolones ciprofloxacin in particular - were used for treating HIV-related diarrhoea and sexually transmitted infections, and an increase in resistance to this drug was observed. Unfortunately this was one of the second-line drugs used for MDR TB and its loss resulted in XDR TB (defined as resistance to rifampicin, isoniazid, any of the aminoglycosides and a fluoroquinolone).

\section{New diagnostic algorithms}

'We've now revised the diagnostic algorithms for TB so that we can identify the high-risk groups early on and test right from the beginning for MDR and subsequently XDR. The highrisk groups are new patients who after 2 months of proper treatment are still TB positive, all the re-treatment patients and all contacts of MDR/XDR TB patients,' she said.

'There is also a need to embark on a nationwide treatment literacy campaign not only for TB but for all chronic diseases, mainly diabetes and hypertension - people just don't seem to get the implications to their lives of not controlling these medical conditions,' she said.

Chris Bateman 